THE ASTROPHYSICAL JOURNAL, 459:653-665, 1996 March 10

C) 1996. The American Astronomical Society. All rights reserved. Printed in U.S.A

$$
1 V+1, u)-3.9
$$

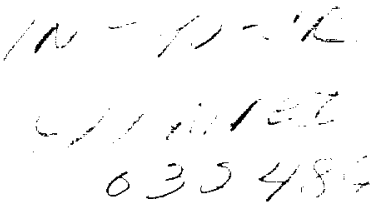

NASA-CR-204588

\title{
AXISYMMETRIC TWO-DIMENSIONAL COMPUTATION OF MAGNETIC FIELD DRAGGING IN ACCRETION DISKS
}

\author{
MAURICIO ReYES-Ruiz ${ }^{1}$ \\ Department of Space Physics and Astronomy, Rice University, Houston, TX 77051 \\ AND \\ TOMASZ F. STEPINSKI \\ Lunar and Planetary Institute, 3600 Bay Area Boulevard, Houston, TX 77058 \\ Received $1995 \mathrm{May} 10$; accepted 1995 September 20
}

\begin{abstract}
In this paper we model a geometrically thin accretion disk interacting with an externally imposed, uniform, vertical magnetic field. The accretion flow in the disk drags and distorts field lines, amplifying the magnetic field in the process. Inside the disk the radial component of the field is sheared into a toroidal component. The aim of this work is to establish the character of the resultant magnetic field and its dependence on the disk's parameters. We concentrate on $\alpha$-disks driven by turbulent viscosity. Axisymmetric, two-dimensional solutions are obtained without taking into account the back-reaction of the magnetic field on the structure of the disk. The character of the magnetic field depends strongly on the magnitude of the magnetic Prandtl number, $\mathscr{P}$. We present two illustrative examples of viscous disks: a so-called "standard" steady state model of a disk around a compact star (e.g., cataclysmic variable), and a steady state model of a proto-planetary disk. In both cases, $\mathscr{P}=1, \mathscr{P}=10^{-1}$, and $\mathscr{P}=10^{-2}$ scenarios are calculated. Significant bending and magnification of the magnetic field is possible only for disks characterized by $\mathscr{P}$ of the order of $10^{-2}$. In such a case, the field lines are bent sufficiently to allow the development of a centrifugally driven wind. Inside the disk the field is dominated by its toroidal component. We also investigate the dragging of the magnetic field by a nonviscous protoplanetary disk described by a phenomenological model. This scenario leads to large distortion and magnification of the magnetic field.
\end{abstract}

Subject headings: accretion, accretion disks - MHD

\section{INTRODUCTION}

Accretion disks are believed to surround many stars. Some of these disks form around compact stars such as white dwarfs, neutron stars, or black holes that are members of binary systems. They reveal themselves as a power source, especially in the X-ray and gamma regions of the spectrum. On the other hand, protostellar disks are believed to be accretion disks associated with young, premain-sequence stars and manifest themselves mostly in infrared and radio observations. These latter disks are considered to be a natural outcome of the star formation process. Furthermore, AGNs are believed to be a manifestation of disk accretion onto a supermassive black hole. Regardless of the astronomical context, accretion disk theory is beset by uncertainty as to the nature of the angular momentum transport mechanism responsible for the accretion of mass onto the central object. Most of accretion disk theory hinges on the notion that accretion is achieved by means of turbulent viscosity. This assumption is based primarily on the very large value of the Reynolds number, which can be as high as $10^{14}$ for disks around compact stars. The actual source of such turbulence is difficult to pinpoint, however. The most obvious candidate, differential rotation, has been largely disregarded as a possible source of turbulence because the Keplerian rotation shear is stable with respect to linear, infinitesimal perturbations. However,

\footnotetext{
1 Postal address : Observatorio Astronomico de Nacional, Apdo. Postal 877, Codigo Postal 22860 Ensenada, B.C., Mexico.
}

it may be unstable with respect to nonlinear, finite am i] tude perturbations (Dubrulle 1992). Another candidate : convection driven by a superadiabatic temperature gradi $n$ across the disk, although it is uncertain whether sucl: gradient can be maintained throughout a signific:1 portion of the disk. Balbus \& Hawley (1991) have show that the presence of a weak magnetic field can shar ? destabilize a Keplerian disk. The resulting magncic rotational instability is currently considered the best cand date for the elusive source of disk turbulence, at least $i$. highly ionized disks where the ideal MHD assumpti , crucial for the existence of such instability, is fulfilled. $\mathrm{In}$ : works for small, hot, and thermally ionized disks arou $n$ compact objects, but is quite problematic for large prot ss tellar disks, which are too cool to be thermally ionized.

Problems with turbulent viscosity have motiva e research on alternative means of accretion. In particu 1 attention has been focused on the effect of an MHD win emanating directly from a disk. Blanford \& Payne 19.3 were the first to argue that such a wind can be so efficien i removing angular momentum as to render turbulent $i$ : cosity unnecessary inasmuch as the existence of an acc $r$ tion process is concerned. Pelletier \& Pudritz (1992) shove that the ratio of the wind torque to the viscous torque o : fluid element of a disk located at a radius $R$ is given $b$ $\left(B_{Z}^{2} / 4 \pi P\right)\left(R_{\mathrm{A}} / \alpha H\right)$. (The list of all symbols used in this pa $x$ is given in Table 1). Typically $R_{\mathrm{A}} / R=3-5$ and, for $\mathrm{g}$ : metrically thin disks, wind torque can indeed be domin: a The hypothesis of wind as an accretion agent gained it dence as it became clear from observations that bipcla 
TABLE 1

Summary of Notation USEd IN THE PAPER

\begin{tabular}{|c|c|}
\hline Symbol & Description \\
\hline 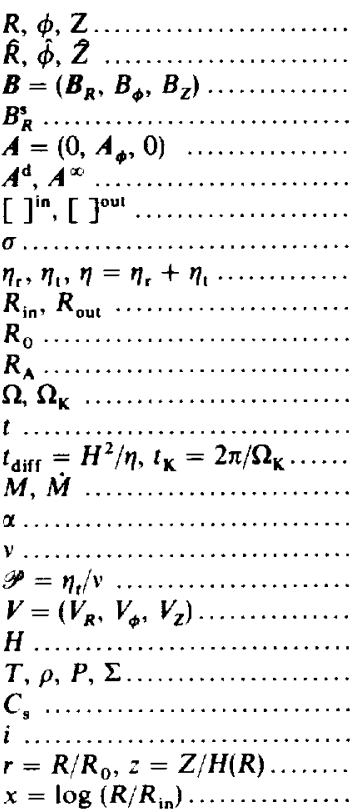 & $\begin{array}{l}\text { Cylindrical polar coordinates } \\
\text { Unit vectors in principal directions } \\
\text { Magnetic field } \\
\text { The value of } B_{R} \text { at the surface of the disk } \\
\text { Vector potential of a poloidal field } \\
\text { Vector potential due to currents in a disk and at infinity } \\
\text { Superscripts "in" and "out " mean inside and outside a disk } \\
\text { Electric conductivity } \\
\text { Resistive, turbulent, and total magnetic diffusivities } \\
\text { Inner and outer radii of a disk } \\
\text { Arbitrary unit of length } \\
\text { Alfvén radius of flow beginning from the disk at } R \\
\text { Angular velocity, Keplerian angular velocity } \\
\text { Time } \\
\text { Diffusion time, Kepler time } \\
\text { Mass of the central star, an accretion rate } \\
\text { Dimensionless turbulent viscosity } \\
\text { Turbulent viscosity } \\
\text { Magnetic Prandtl number } \\
\text { Velocity field } \\
\text { Disk's half-thickness } \\
\text { Temperature, density, pressure, surface density at the midplane } \\
\text { Speed of sound } \\
\text { Angle between a field line and the normal to the disk } \\
\text { Dimensionless spatial variables } \\
\text { Logarithmically spaced radial variable }\end{array}$ \\
\hline
\end{tabular}

outflows (winds or phenomena believed to be driven by winds) are commonly associated with young stellar objects, AGNs, X-ray binaries, and perhaps cataclysmic variables (see review by Pringle 1993).

For a wind to be effective in removing angular momentum from the disk, it must be magnetized. The magnetic field must have a global character and a proper geometry, with open field lines that should make a sufficiently large angle, $i$, with the normal to the disk. According to Blanford \& Payne (1982), assuming cold disk plasma, $i>30^{\circ}$ is required. Are accretion disks magnetized and, if so, is the geometry needed for centrifugally driven winds to occur feasible? Although presently there is no direct observationai evidence for magnetic fields in accretion disks (however, see Horne 1994), we expect disks to be magnetized. Scenarios proposed for magnetization of accretion disks can be broadly divided into two distinct categories: internally generated field and externally maintained field. An internally generated field results from a self-excited dynamo process that builds up a magnetic field from an arbitrarily small seed field. This can be achieved by either a turbulent dynamo (Pudritz 1981; Stepinski \& Levy 1991) or a process involving a magnetorotational instability (Tout \& Pringle 1992). In general, conditions in most accretion disks are favorable for internal generation. Although the major theoretical attraction of self-excited processes is their independence from any external magnetic field, they will amplify such a field if present. For example, Curry, Pudritz, \& Sutherland (1994) showed that a global magnetorotational instability acting in an accretion disk permeated by an external magnetic field could amplify the field and may modify its large-scale structure. If we ignore the effects of self-generating processes then an externally maintained field results from dragging inward an externally imposed field. In such a scenario the source of magnetic field amplification is the radial velocity. This mechanism has received a lot of attention in connection with wind phenomena because the resultant structure of the magnetic field (dipole-like symmetry, open field lines) seems to be favorable for launching centrifugally driven winds. Conversely, internally generated fields are expected to have quadrupole-like symmetry with closed field lines (see Stepinski \& Levy 1991), a configuration that does not favor the initiation of wind.

In the present work, we investigate the configuration of the magnetic field in and around the disk resulting from dragging an initially uniform externally maintained field by a prescribed accretion flow. We stress that the prolonged existence of such a field is possible only if accompanied by the concomitant existence of a magnetic field at "infinity." If the currents supporting the magnetic field at infinity dwindle, so does the magnetic field in a disk. The astronomical context of an external field is not immediately clear, with the possible exception of young stellar objects where an external field can be identified with the magnetic field permeating the remnant molecular cloud. Our motivation is to establish under what conditions, if at all, the dragging of an external field will distort field lines to such an extent as to allow centrifugally driven winds to develop. Our calculations are not self-consistent; we consider the structure of the disk to be given and not affected by the magnetic field. There is no wind present in our approach, so accretion is realized by either turbulent viscosity or, in one particular example, by unspecified means. Thus, our calculations are aimed at studying the onset of winds, rather than a hypothetical disk driven by a fully developed wind.

Because the ability of the accretion flow to bend field lines is so critical for the viability of centrifugally driven winds, the problem we embark on solving has been considered before, although not in the most direct and general fashion. Calculations carried out by van Ballegooijen (1989) addressed the problem of axisymmetric magnetic field amplification by accretion flow in the context of cataclysmic 
variables. His model lacked any externally imposed field, probably because the source of such a field is not apparent in close binaries, although either of the two stars constituting the system can be a potential source of an external field, a possibility explicitly discarded by van Ballegooijen. Without an external field he found that a magnetic field anchored in the disk decays, an expected result in light of a general theorem proven by Cowling (1934) who had shown that an axisymmetric magnetic field cannot be sustained by the motion of the fluid occurring in a bounded volume. Recently, Lubow, Papaloizou, \& Pringle (1994a) revisited the van Ballegooijen problem, but with the addition of an externally generated field. In this case Cowling's theorem does not apply as there exist currents at infinity that maintain an external field, so the problem is, strictly speaking, defined in an unbounded volume. They found that indeed the magnetic field is sustained and a steady state configuration of the magnetic field is reached. Furthermore, they established that the character of a so-engendered magnetic field depends upon a single, dimensionless parameter $\mathscr{D}=$ $2 \eta /\left(3 H\left|V_{R}\right|\right)$. The all-important value of the angle $i$ relates to $\mathscr{D}$ by the relationship $\tan i=1.52 \mathscr{D}^{-1}$. Lubow et al. proffer these results as general enough to be applicable to a comprehensive model of disk evolution, one in which a centrifugally driven wind is responsible for the accretion flow, and the flow, in turn, bends the magnetic field enabling the wind. However, the method of solution employed by Lubow et al. is quite simplified, and it is not clear whether their solution capture the essential feature of the problem. Following von Ballegooijen, they consider what is, in effect, a one-dimensional problem inasmuch as they vertically average the hydromagnetic equation inside the disk. As a result, the structure of the magnetic field inside the disk has to be assumed instead of calculated. The discussion of their solutions is docile by virtue of being dependent upon a single parameter $\mathscr{D}$. This, however, is an artifact of not only one-dimensionality, but also of a particularity of their disk model, which assumes that the disk half-thickness, $H$, depends linearly on the distance from the star, $R$, and that magnetic diffusivity is uniform throughout the disk. Although such assumptions are quite sensible for accretion disks around compact stars, they are inadequate for other classes of accretion disks. In particular, physical conditions describing the state of accretion disks around young, premain-sequence stars are incompatible with Lubow et al. model. Considering the importance of this problem for the viability of centrifugally driven winds, and its ramifications for accretion disk theory, we decided to take a more general approach to the problem of magnetic field dragging in accretion disks.

Our adopted approach is based on the following assumptions: (1) the axisymmetric solution of the full hydromagnetic equation in the entire unbounded space; (2) the existence of an external field maintained by currents located at infinity; (3) a kinematic approach in which all properties of the disk are given a priori and remain unchanged; (4) a vacuum exists outside the disk $(|Z|>H)$; (5) inside the disk all quantities, except the calculated magnetic field, are vertically uniform, (6) magnetic fields are not amplified by either a turbulent dynamo or the magnetorotational instability; and (7) magnetic losses are dominated by turbulent and resistive (ohmic) dissipation. Note that the first assumption aims at obtaining the solution outside and inside the disk concurrently, and the third assumption implies that any steady state model of an accretion disk can be consid $\mathrm{r}$ : using our approach. The assumption of a vacuum out is the disk is made reluctantly for the sole purpose of $\sin p$. city. We do not expect that the space outside the dis: indeed a vacuum; instead we expect that the motion of $1:$ diluted matter there is controlled by the magnetic fiel 1 contrast to the inside of the disk where the behavior of $t$. magnetic field is ruled by the motion of the gas. In o $h_{i}$ words, we expect the magnetic field outside the disk to i almost force-free. As finding a general configuration f force-free magnetic field is beyond the scope of this pa $x$ we assume the simplest force-free pattern given 1 $\nabla \times B=0$, which also happens to define a magnetic fiel vacuum. The assumptions about ignoring the generating processes follows from our desire to isolate $\mathrm{tl}$ consequences of field dragging. The last assumption $\mathrm{el} m$ nates from our considerations cold disks with such 0 density that magnetic losses are primarily due to ambip $]$ diffusion. Such conditions may exist in very extended (sc 1 . of hundreds of $\mathrm{AU}$ ) parts of disks around young stars, ith bending of magnetic fields under these circumstances $v$ : considered by Königl (1989) and Wardle \& Königl (1993,

Our method of finding the magnetic field configuration described in $\S 2$. The next three sections are devoted i particular disk models. In $\S 3$ we apply our method to it so-called "standard" model for thin accretion disks aro $n$ compact stars, and in $\S 4$ we employ our method fo: fiducial model of a proto-planetary disk. These are $b, t$ viscous disks, and we have found that viscous disks a significantly bend magnetic field lines only under scra questionable physical assumptions. Thus, in $\S 5$ we consi $1 \mathrm{c}$ a phenomenological model of a proto-planetary disk th: $t$ characterized by a swift inward flow and is, therefor capable of significantly bending field lines. Finally, in $\$ 6 \mathrm{u}$ summarize our findings and discuss some implications , our results.

\section{EQUATIONS OF THE MODEL}

We consider the interaction of an inward flow of mate is inside a disk with an externally imposed magnetic field. Th notation used throughout the paper is summarized in Ta ol 1. Cylindrical polar coordinates are used, with $Z=0$ corre sponding to the disk midplane and $R=0$ corresponding $t$ the center of the star. In the nonrelativistic case the Maxu e. equations complemented by Ohm's law give the hyd: magnetic (induction) equation that determines the behavic of a magnetic field.

$$
\frac{\partial B}{\partial t}=\nabla \times(V \times B)-\nabla \times(\eta \nabla \times B)
$$

Because the system is axisymmetric, we can express $h$ magnetic field in the form $\boldsymbol{B}=B_{\phi} \hat{\phi}+\nabla \times A_{\phi} \hat{\phi}$. The te $r$ $B_{\phi}$ represents the toroidal field and the term $\nabla \times A_{\phi} \hat{\phi} \mathrm{r}: \mathrm{p}$ resents a poloidal field with components $B_{R}=-\partial A_{\phi} / \bar{l}$ and $B_{Z}=(1 / R) \partial / \partial R\left(R A_{\phi}\right)$. Under such an assumpti in: induction equation (1) is reduced to a set of two sca.a equations:

$$
\begin{aligned}
\frac{\partial B_{\phi}}{\partial t}=-R\left[\frac{\partial \Omega}{\partial R}\right] \frac{\partial A_{\phi}}{\partial Z}+\eta\left[\frac{\partial}{\partial R} \frac{1}{R}\right. & \left.\frac{\partial}{\partial R}\left(R B_{\phi}\right)\right] \\
& +\eta \frac{\partial^{2} B_{\phi}}{\partial Z^{2}}-\frac{\partial\left(V_{R} B_{\phi}\right)}{\partial R}
\end{aligned}
$$


and

$$
\begin{aligned}
\frac{\partial A_{\phi}}{\partial t}=\eta\left[\frac{\partial}{\partial R} \frac{1}{R} \frac{\partial}{\partial R}\left(R A_{\phi}\right)\right]+\eta \frac{\partial^{2} A_{\phi}}{\partial Z^{2}} & \\
& -\frac{V_{R}}{R} \frac{\partial}{\partial R}\left(R A_{\phi}\right) .
\end{aligned}
$$

Several terms have been omitted in deriving equations (2) and (3) from the general form of induction equation (1). First, we neglect the vertical component of the velocity field because in geometrically thin disks, to which we want to apply our calculations, $V_{Z} \ll V_{R} \ll V_{\phi}$. However, we keep the radial component of the velocity field, as it is the only source of magnetic field amplification in our model. Second, we neglect a term in equation (2) proportional to $\partial \eta / \partial R$ because in a thin disk this term is small in comparison with terms proportional to the magnetic diffusivity itself. Note that equation (3), governing the evolution of the poloidal field, is completely decoupled from equation (2), governing the behavior of the toroidal field. This is a consequence of the kinematic approximation and the omission of any selfgeneration mechanisms such as a turbulent dynamo. Therefore, we can first solve for the poloidal field (eq. [3]) and later find the toroidal field (eq. [2]) using the previously obtained solution for the poloidal field. Note, that in such an approach, the poloidal field structure is not affected by the toroidal field.

Equations (2) and (3), describing the evolution of a magnetic field inside a disk, must be supplemented by equations ruling the behavior of a magnetic field outside a disk, which are easily obtainable under our assumption of a vacuum exterior. The toroidal magnetic field outside a disk must vanish because of assumed axisymmetry. As a vacuum is current free, the poloidal field satisfies Ampere's law, $\nabla \times B=\nabla \times \nabla \times A \hat{\phi}=0$, which has an explicit form

$$
\frac{\partial}{\partial R}\left[\frac{1}{R} \frac{\partial}{\partial R}\left(R A_{\phi}\right)\right]+\frac{\partial^{2} A_{\phi}}{\partial Z^{2}}=0 .
$$

The magnetic field must be continuous at the disk surfaces, imposing certain boundary conditions for $A_{\phi}^{\text {in }}( \pm H)$ and $A_{\phi}^{\text {out }}( \pm H)$. At infinity the field must be identical to the external magnetic field. Our models of accretion disks have finite radial extent, limited by an inner radius, $R_{\mathrm{in}}$, and an outer radius, $R_{\text {out }}$. We assume that the vector potential at both radial boundaries is equal to the vector potential of the external field. Finally, we force our solution to have a dipole symmetry by requiring that the radial component of the magnetic field vanish at the disk midplane. The summary of all boundary conditions is given in Table 2 .

TABLE 2

Summary of Magnetic Field Boundary Conditions

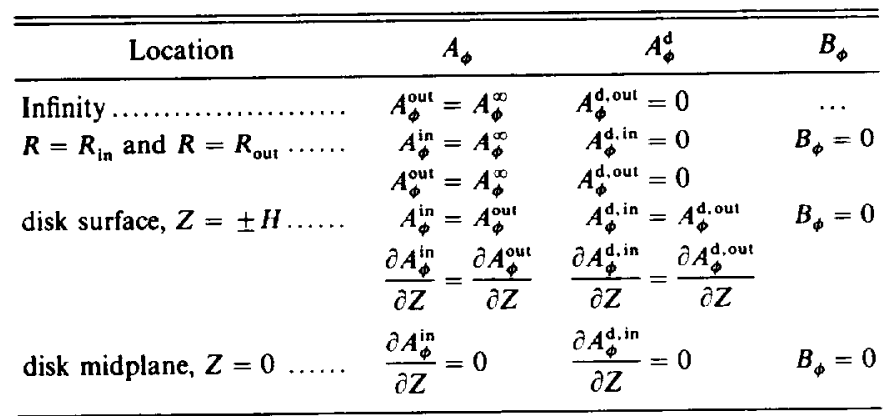

The dependence of the poloidal field on an externally imposed magnetic field is implicit in equation (3), as it enters through boundary conditions. It is desirable, both for computational reasons and for greater clarity, to have the driving equation explicitly dependent on the external field. This may be achieved by splitting the magnetic potential into a contribution from currents within a disk, $A_{\phi}^{\mathrm{d}}$, and the potential from currents located at infinity, $A_{\phi}^{\infty}$, so

$$
A_{\phi}=A_{\phi}^{\mathrm{d}}+A_{\phi}^{\infty} \text {. }
$$

We now assume that the externally imposed magnetic field is uniform and directed along $\hat{Z}, B^{\infty}=\left(0,0, B^{\infty}\right)$, with $B^{\infty}$ uniform and given. The magnetic potential of such a field is $A_{\phi}^{\infty}=B^{\infty} R / 2$. Substituting representation (5) into equations (3) and (4) we obtain

$$
\begin{aligned}
\frac{\partial A_{\phi}^{\mathrm{d}, \mathrm{in}}}{\partial t}=\eta\left[\frac{\partial}{\partial R} \frac{1}{R}\right. & \left.\frac{\partial}{\partial R}\left(R A_{\phi}^{\mathrm{d}, \mathrm{in}}\right)\right] \\
& +\eta \frac{\partial^{2} A_{\phi}^{\mathrm{d}, \mathrm{in}}}{\partial Z^{2}}-\frac{V_{R}}{R} \frac{\partial}{\partial R}\left(R A_{\phi}^{\mathrm{d}, \mathrm{in}}\right)-V_{R} B^{\infty}
\end{aligned}
$$

inside the disk, and

$$
\frac{\partial}{\partial R}\left[\frac{1}{R} \frac{\partial}{\partial R}\left(R A_{\phi}^{\mathrm{d}, \text { out }}\right)\right]+\frac{\partial^{2} A_{\phi}^{\mathrm{d}, \text { out }}}{\partial Z^{2}}=0
$$

outside the disk. Equations (6) and (7) are supplemented by the boundary conditions given in the third column of Table 2. Terms on the right-hand side of equation (6) represent radial diffusion of the poloidal field, the diffusive drift of the poloidal field out of the disk (vertical diffusion), advection of the poloidal field with the accretion flow, and the source associated with the external field, respectively. As the magnetic field inside the disk evolves, so does the field outside the disk; even so, there is no explicit time dependence present in equation (7). The time dependence enters into the solution of equation (7) through boundary conditions at disk surfaces. The solution of equations (6) and (7), subject to the boundary conditions shown in Table 2 , constitute the principal problem of this paper.

Let us once again enumerate the steps we perform to find the total structure of a magnetic field resulting from dragging of an external field. First we solve the system of equations (6) and (7) to find $A_{\phi}^{\mathrm{d}}$ inside and outside the disk. We add the result to $A_{\phi}^{\infty}$, thereby obtaining the total potential of the poloidal magnetic field, $A_{\phi}$. Components of the poloidal field, $B_{R}$ and $B_{Z}$, are now obtainable from the formulas given just above the equation (2), whereas the poloidal field lines are calculated from the relation $R A_{\phi}=$ constant. Finally, the toroidal field, $B_{\phi}$, is calculated from equation (2), subject to the boundary conditions given in the last column of Table 2 . This concludes the description of the approach we have taken to calculate the outcome of magnetic field dragging in accretion disks. The remainder of this section recounts certain technical details of our approach and its numerical implementation.

Because we want to reckon with steady state models of thin accretion disks that otherwise are quite general, we need to transform our equations so they can be defined on a rectangular spatial domain. Let us introduce new spatial coordinates, $r=R / R_{0}$ and $z=Z / H(R)$. Thus, we would measure radial distance in some arbitrary unit $R_{0}$ (not necessarily equal to either $R_{\text {in }}$ or $R_{\text {out }}$ ), and vertical distance in 
units of the local half-thickness of the disk. The transformation $(R, Z) \rightarrow(r, z)$ brings equation (6) into the following form:

$$
\begin{aligned}
\frac{\partial A_{\phi}^{\mathrm{d}, \text { in }}}{\partial t}=\frac{\eta}{R_{0}^{2}} & {\left[\frac{\partial}{\partial r} \frac{1}{r} \frac{\partial}{\partial r}\left(r A_{\phi}^{\mathrm{d}, \text { in }}\right)\right] } \\
& +\frac{\eta}{H^{2}} \frac{\partial^{2} A_{\phi}^{\mathrm{d}, \text { in }}}{\partial z^{2}}-\frac{V_{R}}{R_{0}} \frac{1}{r} \frac{\partial}{\partial r}\left(r A_{\phi}^{\mathrm{d}, \text { in }}\right)-V_{R} B^{\infty} .
\end{aligned}
$$

Now equation (8) is defined on a rectangular domain $r \in$ $\left(R_{\mathrm{in} /} / R_{0}, R_{\text {out }} / R_{0}\right), z \in(0,1)$. The domain of a vertical coordinate starts at the midplane $(z=0)$ because, due to assumed dipole symmetry, it is sufficient to consider only the space above the midplane. Note, however, that the transformation $r=R / R_{0}$ and $z=Z / H(R)$ leaves equation (7) still in the unbounded domain as $Z \in(H, \infty)$ transforms into $z \in(1, \infty)$. Therefore, such a transformation is inappropriate for the space outside the disk. However, a different transformation $r=R / R_{0}$ and $z=H(R) / Z$ happens to map the outside of the disk into the same rectangle on which equation (8) is defined. The new form of equation (7) is

$$
\begin{aligned}
{\left[\frac { \partial } { \partial r } \frac { 1 } { r } \frac { \partial } { \partial r } \left(r A_{\phi}^{\text {d,out })]+\frac{R_{0}^{2}}{H^{2}} z^{4} \frac{\partial^{2} A_{\phi}^{\text {d,out }}}{\partial z^{2}}}\right.\right.} \\
+2 \frac{R_{0}^{2}}{H^{2}} z^{3} \frac{\partial A_{\phi}^{\text {d,out }}}{\partial z}=0 .
\end{aligned}
$$

Both equations (8) and (9) are defined on the same finite, rectangular spatial domain, and are coupled by means of boundary conditions at $z= \pm 1$. The total set of boundary conditions for the redefined problem (8) and (9) is almost identical to those listed in the third column of Table 2 if $R$ and $Z$ are replaced by $r$ and $z$. The only difference is the second condition at the surface of the disk $(z= \pm 1)$, which now reads $\partial A_{\phi}^{\mathrm{d}, \text { in }} / \partial z=-\partial A_{\phi}^{\mathrm{d}, o u t} / \partial z$. Finally, to have a better resolution in the radial direction, we change $r$ to a logarithmical spaced variable $x$ according to $r=\left(R_{\mathrm{in}} / R_{0}\right) 10^{x}$. As this changes only a radial variable, the form of boundary conditions is unaffected by such a transformation.

Two different numerical methods are employed to solve the set of equations (8) and (9). The physics of the problem hints at the existence of a steady state solution when the amplification of the field by inward dragging is balanced by magnetic diffusion. Thus, our first numerical method is based on an assumed steady state. We put the time derivative to zero and discretize the spatial domain with an even spacing mesh in both coordinates $x$ and $z$. The resulting set of finite differences equations constitutes a set of linear algebraic equations, which is solved using the Gauss elimination method. We use this method for the bulk of our calculations. However, to obtain information about the time necessary to achieve such a steady state, a second method, utilizing time-dependent calculations, is used. We discretize our equations using finite differences, first-order in time and second-order in space. Inside the disk we solve the resultant set of finite differences equations by the Euler method. The equation describing the magnetic potential outside the disk is solved with a simple relaxation scheme. After the inside equation is advanced one time step, we iterate the outside equation until the boundary conditions at the disk surface are met. Both methods eventually the same solution.

\section{MAGNETIC FIELD DRAGGING IN THE STANDARI ACCRETION DISK MODEL}

In this section we examine the magnetic field generate I a result of dragging an external field by matter in our s called standard accretion disk with parameters that rou $\mathrm{h}$ correspond to those in cataclysmic variables. $\mathrm{N}$ ? however, that because this work is geared toward un ic standing the basic features of magnetic field draggin: accretion disks, our choice of disk model is dictated b: simplicity rather than by how well it describes the phe : mena of cataclysmic variables. The steady state modei such a disk was first investigated by Shakura \& Suny $t$ (1973), and it is based on the $\alpha$ prescription of viscosity. $\forall$ assume a disk surrounding a compact nonmagnetic t with mass $M=1 M_{\odot}$ and a radius of about $10^{9} \mathrm{~cm}$. n disk extends from about the star surface $\left(R_{\text {in }}=10^{9} \mathrm{~cm}\right)$ $R_{\text {out }}=10^{12} \mathrm{~cm}$. The typical temperatures are from 10 near the inner radius to $10^{3} \mathrm{~K}$ near the outer radius. $\mathrm{T}$ ! , thermal ionization is high and resistive diffusivity, of il order of $10^{4}-10^{7} \mathrm{~cm}^{2} \mathrm{~s}^{-1}$, is negligible in comparison to turbulent magnetic diffusivity, which is of the order of $1(1$ : $10^{14} \mathrm{~cm}^{2} \mathrm{~s}^{-1}$. A characteristic magnetic field diffusion $\mathrm{t} \mathrm{r}$ : $t_{\text {diff }} \sim H^{2} / \eta$, is of the order of $10^{2}-10^{4} \mathrm{~s}$, so for a magn $\mathrm{s}$ field to persist it must be either internally generated . amplified from an externally supplied source. The phys $c$ quantities in the standard model are described by (see, : Frank, King, \& Raine 1985)

$$
\begin{aligned}
& H=1.7 \times 10^{8} \alpha^{-1 / 10} \dot{M}_{16}^{3 / 20} M_{1}^{-3 / 8} R_{10}^{9 / 8} \mathrm{~cm} \\
& V_{R}=-2.7 \times 10^{4} \alpha^{4 / 5} \dot{M}_{16}^{3 / 10} M_{1}^{-1 / 4} R_{10}^{-1 / 4} \mathrm{~cm} \mathrm{~s}^{-1}, 1 \\
& \eta_{t}=1.8 \times 10^{14} \mathscr{P} \alpha^{4 / 5} \dot{M}_{16}^{3 / 10} M_{1}^{-1 / 4} R_{10}^{3 / 4} \mathrm{~cm}^{2} \mathrm{~s}^{-1}
\end{aligned}
$$

In the above formulae, the radial coordinate is measure units of $10^{10} \mathrm{~cm}$, the accretion rate is measured in unit $10^{16} \mathrm{~g} \mathrm{~s}^{-1}$, and the mass of the central star is measures units of $M_{\odot}$. Because of our kinematic approximation $I$ $V_{R}$, and $\eta_{t}$ are the only disk quantities needed to calcu $\downarrow$ the configuration of a magnetic field. Substituting them int equation (6) fully determines the problem. Thus, it problem of magnetic field dragging in the standard dis parameterized by the magnetic Prandtl number, $\mathscr{P}$, in at $d$ tion to the disk's usual parameters, $\alpha, \dot{M}$, and $M$. We fol o Lubow et al. (1994a) in defining $\mathscr{P}=\eta_{t} / v$, note, howe $\mathrm{e}$ that such a definition is an inverse of what is convention : 1 used.

The externally imposed magnetic field is uniform . $n$ aligned vertically. We calculate the steady state configi ration of the magnetic field as it is distorted and magni ic by the action of an accretion flow in a standard disk charia terized by $\alpha=0.1, \dot{M}_{16}=1$, and $M_{1}=1$. Figure 1 sho $u$ the summary of the resultant magnetic field properties. ${ }^{\prime}$ l most distinctive feature of the calculated magnetic field $c$, figuration is its strong dependence on the value of $\mathscr{P}$. Fo close to its fiducial value of the order of unity, the exter 1: field is only barely distorted. The magnitude of the vert $c$ : field within a disk remains very close to the value of it: externally imposed field. Both radial and transvi:(toroidal) components of the magnetic field appear wit.ii the disk, but they are about 2 orders of magnitude smailk than the vertical component. Overall, the standard acirt tion disk with fiducial values of its controlling parame:el 

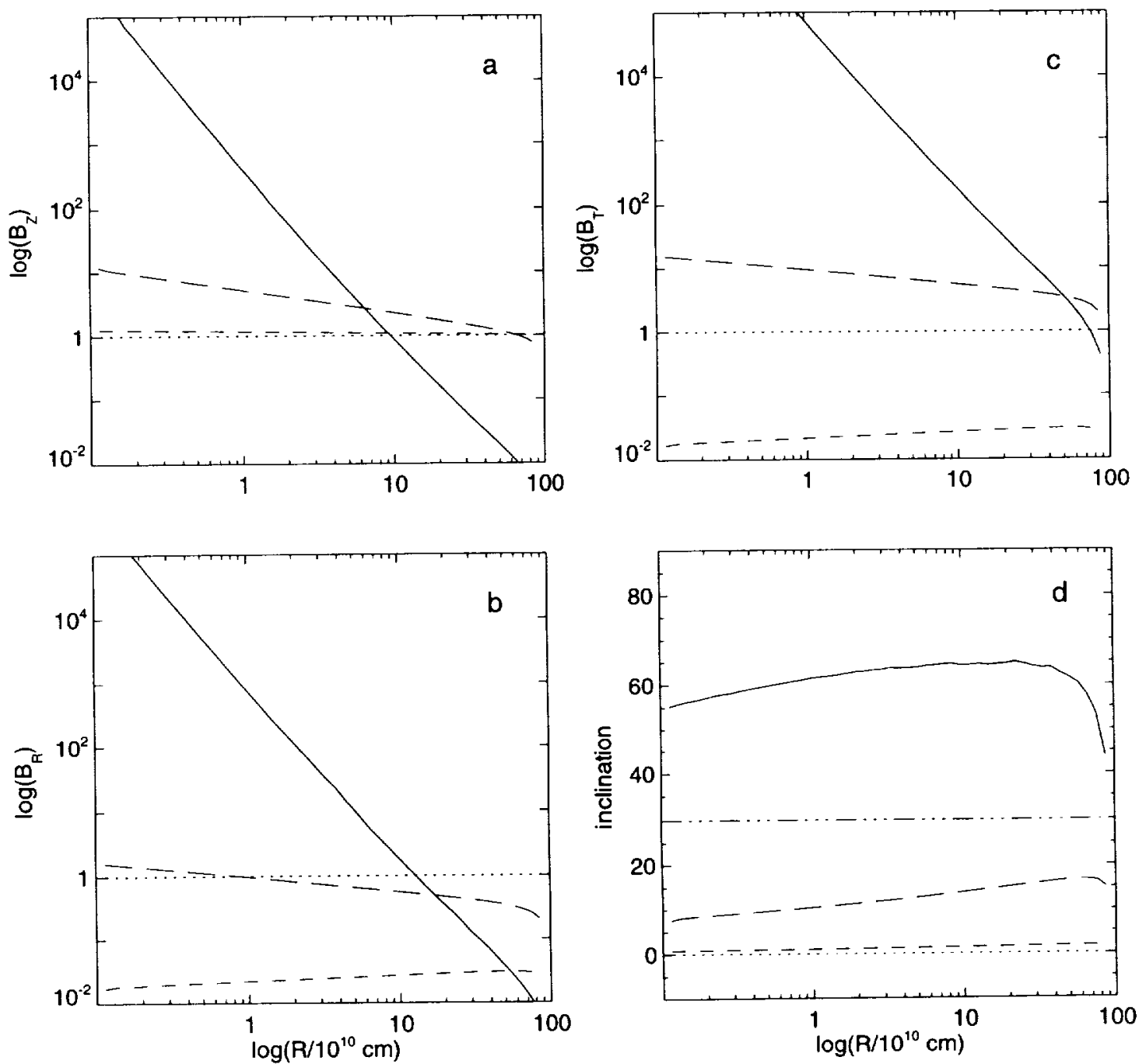

FiG. 1.-Properties of the steady state magnetic field resulting from dragging an initially uniform vertical field by the flow in a standard accretion disk. Different line styles correspond to different values of magnetic Prandtl number: $\mathscr{P}=1$, short-dashed line; $\mathscr{P}=0.1$, long-dashed line; $\mathscr{P}=0.01$, solid line. $(a-c)$ The strength of magnetic fields in a disk as a function of distance from the central star: $(a)$ vertical field at the disk's midplane; $(b)$ toroidal field at the vertical location where it achieves its maximum value; $(c)$ radial field at the surface of the disk. The magnitude of the magnetic field is measured in units of $B^{*} ;$ the dotted lines, drawn for visual reference, indicate $B=B^{\infty}$. (d) Inclination angle, $i$, between the field lines and the normal to the disk measured at the disk surface, as a function of the distance from the star. Dotted and triple-dot-dashed lines are for visual reference indicating $i=0$ and $i=30^{\circ}$, respectively.

has a negligible effect on the external field. The distortion suffered by the field lines is too small to permit any centrifugally driven wind to occur. Moreover, this inability to bend field lines significantly is the prevailing feature of standard disks with $\mathscr{P} \approx 1$. Changing other controlling parameters does not produce a qualitatively different result. A disk's thickness depends only weakly on these parameters (see eq. $[10]$ ), so surface boundary conditions are located at approximately the same place regardless of the values of $\alpha$, $M$, and $M$. Governing equation (6) depends only on the ratio $V_{R} / \eta$, and for a standard disk, where magnetic diffusivity is dominated by turbulence, this ratio is independent of $\alpha, \dot{M}$, and $M$ (see eqs. [11] and [12]).

The features of the poloidal magnetic field can be anticipated from equation (3) if we neglect radial diffusion and assume within the disk $B_{R} \approx B_{R}^{\mathrm{s}}(Z / H)$. Then we have

$$
\frac{B_{R}^{\mathrm{s}}}{B_{\mathrm{Z}}} \approx-\frac{V_{R} H}{\eta} .
$$

In a standard disk, where $\eta \approx \eta_{t}$, we can use equations (11) and (12) to estimate that $B_{R}^{\mathrm{s}} / B_{Z} \approx 1.5(H / R)(1 / \mathscr{P})$. The ratio
$H / R$ is roughly about 0.02 and equation (13) reduces to

$$
\frac{B_{R}^{\mathrm{s}}}{B_{Z}} \approx \frac{3 \times 10^{-2}}{\mathscr{P}} .
$$

We can also anticipate the features of the toroidal magnetic field from equation (2) if we neglect the radial diffusion and advective terms. Under such an approximation equation (2) reduces to $\partial^{2} B_{\phi} / \partial^{2} Z=1.5 B_{R} \Omega_{\mathbb{K}} / \eta$. If we again assume that within a disk $B_{R} \approx B_{R}^{s}(Z / H)$, we can analytically calculate $B_{\phi}$

$$
\frac{B_{\phi}}{B_{R}^{s}}=\frac{1}{4} \frac{\Omega_{\mathrm{K}} H^{2}}{\eta}\left[\left(\frac{Z}{H}\right)^{3}-\frac{Z}{H}\right]
$$

The toroidal field achieves its maximum value at height $Z=(1 / 3) \approx 0.6 \mathrm{H}$ from the midplane. In a standard disk $\eta=\eta_{t}=\mathscr{P} v=\mathscr{P} \alpha \Omega_{\mathbf{K}} H^{2}$ and equation (15) reduces to

$$
\frac{B_{\phi}(Z=0.6 H)}{B_{R}^{\mathrm{s}}} \approx \frac{-0.096}{\alpha \mathscr{P}} .
$$


The inclination angle, $i$, is such that $\tan i=B_{R}^{\mathrm{s}} / B_{Z}$, or using equation (13), $i=\arctan \left(-V_{R} H / \eta\right)$. In a standard disk $i \approx \arctan \left(3 \times 10^{-2} / \mathscr{P}\right)$. Our numerical calculations (see Fig. 1) are in agreement with the above estimations. For $\mathscr{P}=1, B_{Z} \approx B^{\infty 1},\left|B_{R}^{s} / B_{Z}\right| \approx 2-3 \times 10^{-2}$, as predicted by equation (14), and $\left|B_{\phi} / B_{R}^{\mathrm{s}}\right| \approx 1$, as predicted by equation (16). The inclination angle is very small, as predicted above.

For smaller values of $\mathscr{P}$ the distortion of the uniform magnetic field becomes noticeable and it may even get to be quite dramatic. For $\mathscr{P}=0.1$ the vertical field in the disk is amplified by about an order magnitude in the inner disk (less at larger radii). Distortion from an originally vertical magnetic field configuration is large enough to build up a significant radial component of magnetic field in a disk, $\left|B_{R} / B_{Z}\right| \sim 0.1$. The radial field, in turn, gives rise to a toroidal field, $\left|B_{\phi} / B_{Z}\right| \geq 1$. However, the inclination angle $i$ is less than the $30^{\circ}$ needed to launch a wind. We presuppose here that the base of the wind coincides with the disk surface defined earlier as the density scale height. That is not necessarily so, but in thin disks the inclination angle calculated at the surface of the disk should be a good approximation to the value of this angle at the base of the wind.

We get a wind-launching configuration of the magnetic field when we assume $\mathscr{P}=0.01$. In this case a dramatic magnification of the magnetic field occurs. The vertical field in the disk is magnified as much as $10^{4}$ times in the innermost part of the disk. The radial field is about as strong as the vertical field, and the toroidal component of the magnetic field dominates the entire field inside the disk, $\left|B_{\phi} / B_{Z}\right| \sim 10^{2}$. The inclination angle $i \approx 60^{\circ}$, larger than the critical wind-launching angle, is maintained throughout the disk. In both cases, $\mathscr{P}=0.1$ and $\mathscr{P}=0.01$, the calculated structure of the magnetic field is very close to what our rough estimations indicate.

Figure 2 shows the magnetic field lines for the case $\mathscr{P}=0.01$. From the solution of the time-dependent equations we can determine that the time necessary for the magnetic field to reach this equilibrium configuration is about $55 \mathrm{yr}$. This is much longer than the dynamical timescale but comparable to the viscous timescale. For the fiducial value of $\mathscr{P}=1$ the field reaches equilibrium after about $1 \mathrm{yr}$, and for the disk characterized by $\mathscr{P}=0.1$ after about $10 \mathrm{yr}$.
The dependence of the magnetic field structure or magnetic Prandtl number is reminiscent of the beh: $i 1$ found by Lubow et al. (1994a). However, there are : s important qualitative differences between their and " results. As we use a specific model of the accretion disk i thickness of our disk is not explicitly a free parameter a: it in the Lubow et al. calculations. This is why our $\mathrm{mr}$ control parameter is the magnetic Prandtl number, wht their only control parameter is $\mathscr{D}=\mathscr{P} /(H / R)$ (note that $: I$ is a constant in their model). The fact that the proble: : Lubow et al. depends only on one parameter (ours dep $\mathrm{n}$ on $\mathscr{P}, \alpha, \dot{M}$, and $M$, but in principle it depends on $\mathscr{P}$ anc $t$ shape of the disk) is an artifact of their approach. Su $\mathrm{h}$ simple parameterization can only be achieved in a dimensional approximation, using a disk model where :I and $\eta$ are constants, and making some additional comp it tional approximations. The solutions of the magnetic is dragging problem obtained from two-dimensional caic lations cannot be, in general, characterized by a si iz parameter. Nevertheless, in the case of a standard iis where the shape of the disk is basically fixed, $\mathscr{P}$ becomes effect the single parameter setting the character of the $\mathrm{pc}$ l, dal field.

Figure $1 a$ shows that the radial dependence of $B_{Z}$ is characterized by a power-law $B_{Z} \sim R^{-x}$. The power lat index, $\kappa$, depends strongly on the value of $\mathscr{P}$. We can st mate that $\kappa(\mathscr{P})$ is itself well described by power-1a $\kappa \approx 0.05 \mathscr{P}^{-0.87}$. Thus, $\kappa$ is almost inversely proportion: : $\mathscr{P}$, translating into very high sensitivity of magnetic $1 \mathrm{c}$ radial dependence on the value of $\mathscr{P}$. The results of Lul io et al. also indicate power-law-like radial dependence of $B$ However, there are significant departures from a si g power law, making a direct comparison of our results in theirs difficult. Nevertheless, if we take the portion of th disk where a single power law approximates their result $v$ notice a dependence of the power-law index on ? $\kappa \approx 0.26 \mathscr{D}^{-0.83}$, or $\kappa \approx 0.26(R / H)^{-0.83} \mathscr{P}^{-0.83}$, very re $\mathrm{n}$ niscent of what we have found. The difference between it results of Lubow et al. and ours is in the value of the $r$, malizing factor; it is 0.05 for our results but about $0.01 \mathrm{fi}$ their results (we assume $R / H \approx 50$ corresponding to it approximate thickness of our standard disk). This differe " may be attributed to differences between the models, $n$
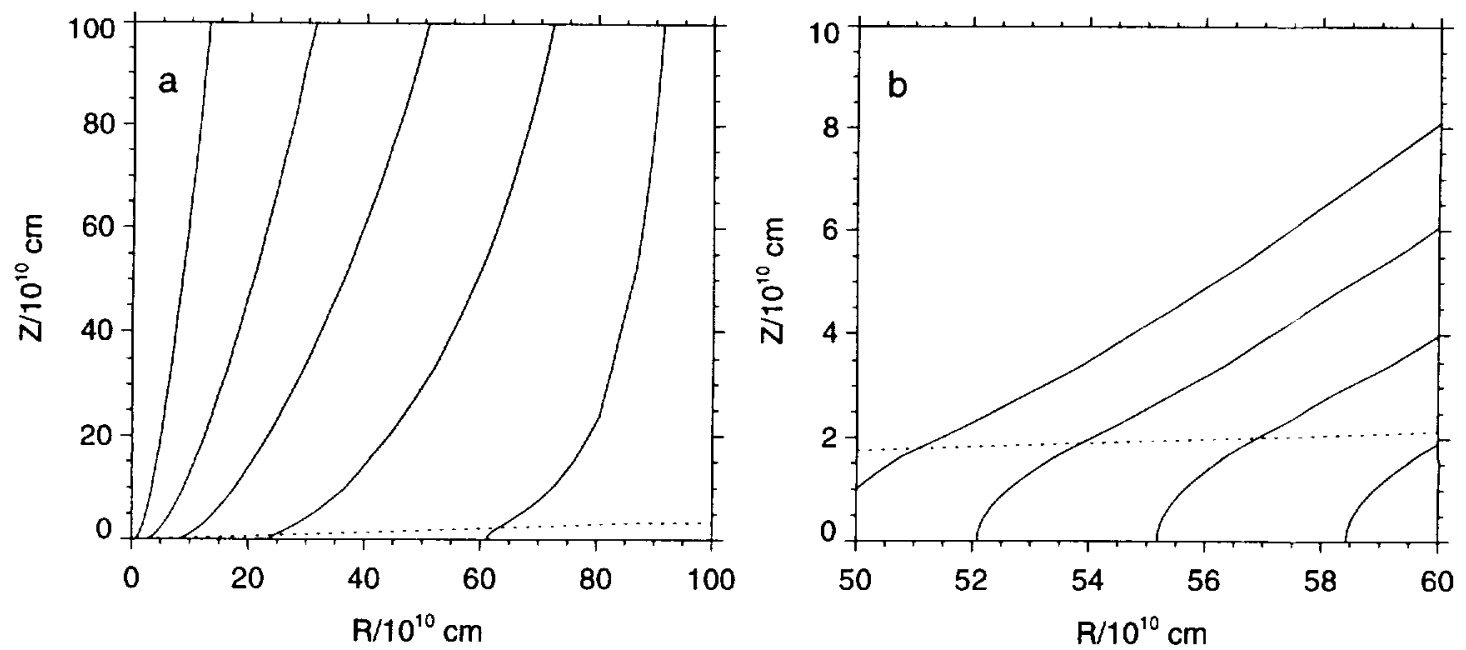

Fig. 2.-Plot of field lines of the steady state poloidal magnetic field resulting from dragging an initially uniform vertical field by the flow in a stand $\mathrm{I}$ accretion disk characterized by $\mathscr{P}=0.01 ;(a)$ global view, (b) close-up of a certain portion of the disk to show the structure of the field inside the disk. 
more likely to our inability to approximate the Lubow et al. results as a single power law. In fact, their Figure 1 shows that the radial dependence of $B_{Z}$ steepens significantly at the inner portion of the disk.

Our calculations reveal the dominance of the toroidal component of the magnetic field in the disk. The toroidal field was ignored by Lubow et al. on the premise that its magnitude depends on the disk thickness and vanishes in the limit of a zero thickness disk (see eq. [15]). However, if magnetic diffusivity is dominated by turbulence, $\eta$ is proportional to $H^{2}$, and although both $B_{R}^{s}$ and $B_{\phi}$ vanish in $H \rightarrow 0$, the ratio $B_{\phi} / B_{R}^{s}$ is independent of the disk thickness (see eq. [16]). In such a disk, the toroidal magnetic field in fact dominates the radial magnetic field, providing that $\alpha \mathscr{P}<0.096$. In addition, a significantly distorted poloidal field (small values of $\mathscr{P}$ ) must be accompanied by a dominant toroidal field, inasmuch as in such a configuration $\left|B_{R} / B_{Z}\right| \sim 1$ and $\left|B_{\phi} / B_{R}\right|$ is large. Note that although the poloidal field is almost independent of the value of $\alpha$, the magnitude of the toroidal field is inversely proportional to $\alpha$.

\section{MAGNETIC FIELD DRAGGING IN VISCOUS PROTO-PLANETARY DISKS}

Astronomical observations of young low-mass stars led to wide acceptance of the idea that these stars are surrounded by accretion disks (for reviews see Strom \& Edwards 1993; Beckwith 1994), often referred to as protoplanetary disks because it is expected that they are progenitors of planetary systems much like our solar system. On the other hand, young stellar objects are capable of producing highly collimated bipolar outflows (for a review see Ray \& Mundt 1993) often explained as a wind centrifugally driven from the disk. Thus, young low-mass stars provide perhaps the most urgent stimulus to study the phenomenon of centrifugally driven wind from disks, inasmuch as all the necessary ingredients are most conspicuously present there.

We assume the proto-planetary disk to be a Keplerian, axisymmetric, geometrically thin, steady state, turbulent $\alpha$-disk surrounding a $1 M_{\odot}$ star and extending up to 100 AU. For the purpose of our calculations, the opacity law, needed to determine the structure of a disk, is taken from Ruden \& Pollack (1991), and the specific formulae for the structure of the steady state disk with this particular choice of opacity are listed in Stepinski, Reyes-Ruiz, \& Vanhala (1993) and Dubrulle (1993). We adopt $\alpha=0.01$ and $\dot{M}=$ $10^{-6} M_{\odot} \mathrm{yr}^{-1}$. These values yield a disk with a mass of about $0.25 M_{\odot}$ and overall properties corresponding to the average $T$ Tauri disk.

The midplane gas temperature is relatively low in extended parts of the proto-planetary disk. The low gas temperature, as well as the high density of gas and dust, result in very low levels of thermal ionization. Therefore, the electrical conductivity in large portions of such a disk is dominated by nonthermal ionization sources of which Galactic cosmic rays are the most important (the others being various radioactive isotopes). The midplane regions of the disk, which interest us most because this is where the bulk of the disk's mass resides, can only be ionized by cosmic rays when the surface density is not high enough to shield them from such ionization. Thus, an interesting pattern of the degree of ionization emerges in protoplanetary disks (Stepinski 1992; Stepinski et al. 1993); the inner, relatively hot part of the disk (in our particular model up to about $3 \mathrm{AU}$ from the star) is highly ionized due to thermal ionization; the outer part of the disk (beyond about $10 \mathrm{AU}$ in our particular model) is ionized to some degree by cosmic rays, but the middle portion of the disk is very weakly ionized because it is too cool for thermal ionization and too dense for cosmic-ray ionization. It is crucial to include both resistive and turbulent magnetic diffusivities when considering magnetic properties of proto-planetary disks because both of them are important, albeit in different radial parts of the disk. We calculate turbulent, $\eta_{t}$, and resistive, $\eta_{\mathrm{r}}$, diffusivities (for details see Stepinski et al. 1993) and use the total diffusivity, $\eta=\eta_{\mathrm{t}}+\eta_{\mathrm{r}}$, in the governing equation (6). Physically, this constitutes the key difference between the case of a proto-planetary viscous disk and the standard disk considered in the previous section.

Figure 3 shows the properties of the magnetic field resulting from the gas dragging of initially uniform field in a proto-planetary viscous disk. The structure of the field exhibits a strong dependence on the magnitude of the magnetic Prandtl number; however, this dependence is most apparent in the inner part of the disk, where magnetic diffusion is completely controlled by turbulence. In fact, the configuration of the magnetic field in the inner part of the proto-planetary viscous disk closely resembles the structure of the magnetic field in the standard disk. Note, however, that $\left|B_{\phi} / B_{R}\right|$ is about an order of magnitude larger in the inner part of the proto-planetary disk than it is in the standard disk. This is because the magnitude of the toroidal field is inversely proportional to $\alpha$, which is an order of magnitude smaller in the proto-planetary disk. In the outer part of the disk the relative importance of resistive and turbulent diffusivity depends on the value of $\mathscr{P}$. For the fiducial value of $\mathscr{P}=1, \eta_{\mathrm{t}}>\eta_{\mathrm{r}}$, but both are of the same order of magnitude. For $\mathscr{P} \ll 1$, magnetic diffusion is dominated by resistive losses. This is why the dependence of magnetic field structure on $\mathscr{P}$, in the outer region of the disk, diminishes as $\mathscr{P}$ decreases. Overall, the electric conductivity in the outer parts of the viscous proto-planetary disk is too low to permit significant bending of an external field, even in the best-case scenario, when turbulence is completely inefficient in reducing the magnetic field (small $\mathscr{P})$. In the middle portion of the disk (3 AU $<R<10 \mathrm{AU})$ where the degree of ionization is very small, resistive diffusion dominates over turbulent diffusion even if $\mathscr{P}=1$. In this region the flow is not able to drag a magnetic field, regardless of how small the value of $\mathscr{P}$ is. Note that the degree of ionization changes sharply from very low to very high near the inner boundary of the low-ionization region as the gas becomes hot enough to ionize alkali metals. This rapid radial change in the magnetic properties of the gas causes the depletion of the vertical field from the part of the disk located immediately inward of the low-ionization region. This is most clearly visible in Figure $3 a$ for $\mathscr{P}=0.01$. The field lines there are advected inward, as they are in a highly conductive medium; however, this is not balanced by the concomitant advection of field lines from the neighboring region as it is ionized too weakly to support any magnetic field advection. Figure 4 shows the magnetic field lines for the case $\mathscr{P}=0.01$.

We calculated the time required for the magnetic field to reach a steady state equilibrium configuration. For $\mathscr{P}=1$ an equilibrium state is reached after $5 \times 10^{3} \mathrm{yr}$. The case with $\mathscr{P}=0.1$ takes $6 \times 10^{3} \mathrm{yr}$ and the case of $\mathscr{P}=0.01$ takes slightly more than $5 \times 10^{4}$ yr to reach a steady state 

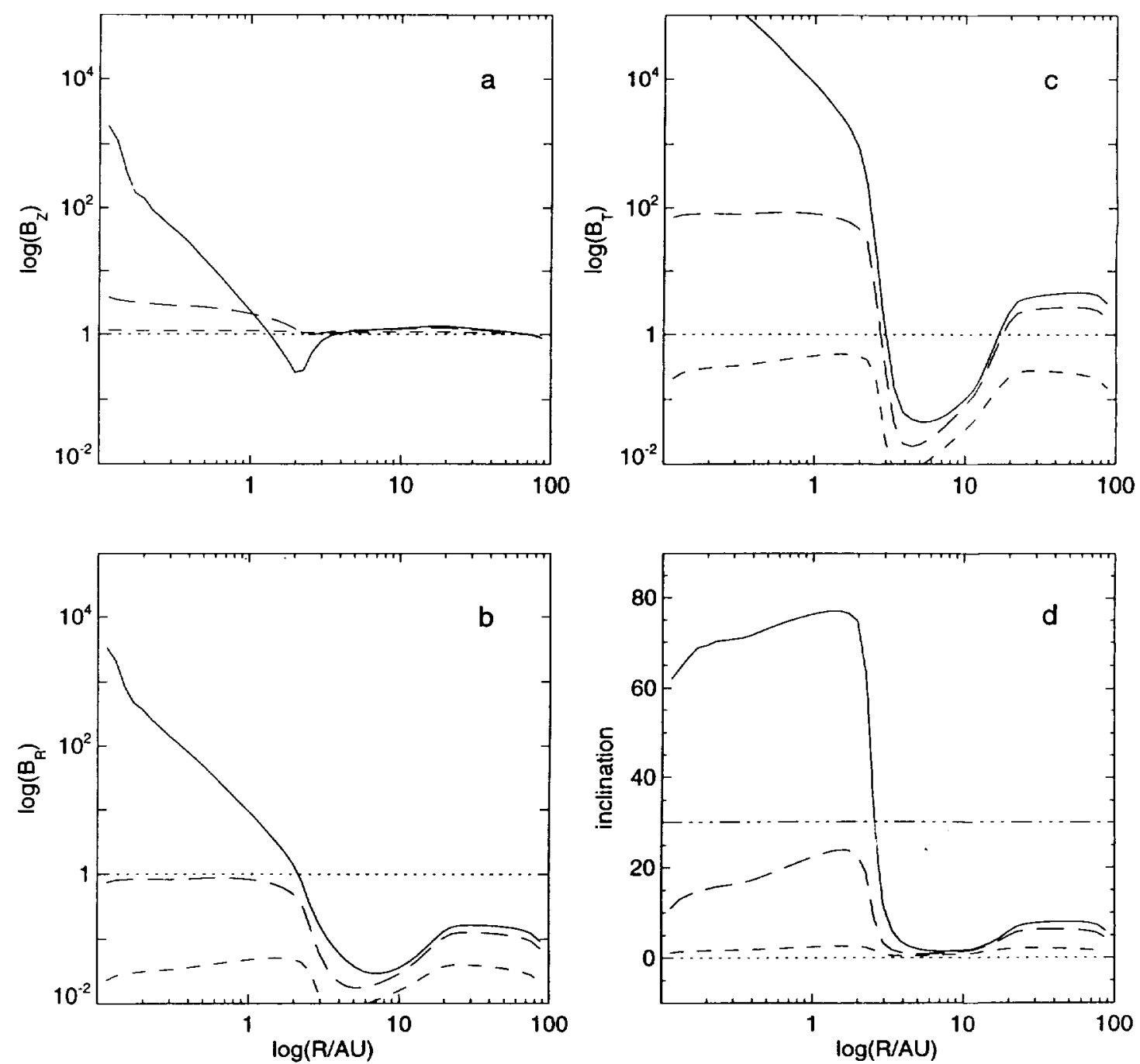

Fig. 3.-Properties of the steady state magnetic field resulting from dragging an initially uniform vertical field by the flow in a viscous proto-plan t. disk. Different line styles correspond to different values of magnetic Prandtl number: $\mathscr{P}=1$, short-dashed line; $\mathscr{P}=0.1$, long-dashed line; $\mathscr{P}=0.01$, soli, $!$ $(a-c)$ The strength of magnetic fields in a disk as a function of distance from the central star: $(a)$ vertical field at the disk's midplane; (b) toroidal field $i: 5$ : vertical location where it achieves its maximum value; $(c)$ radial field at the surface of the disk. The magnitude of the magnetic field is measured in units $c: l i$ the dotted lines, drawn for visual reference, indicate $B=B^{\infty}$. (d) Inclination angle, $i$, between the field lines and the normal to the disk measured at the $\mathrm{d}$ : surface, as a function of the distance from the star. Dotted and triple-dot-dashed lines are for visual reference indicating $i=0$ and $i=30^{\circ}$, respectively
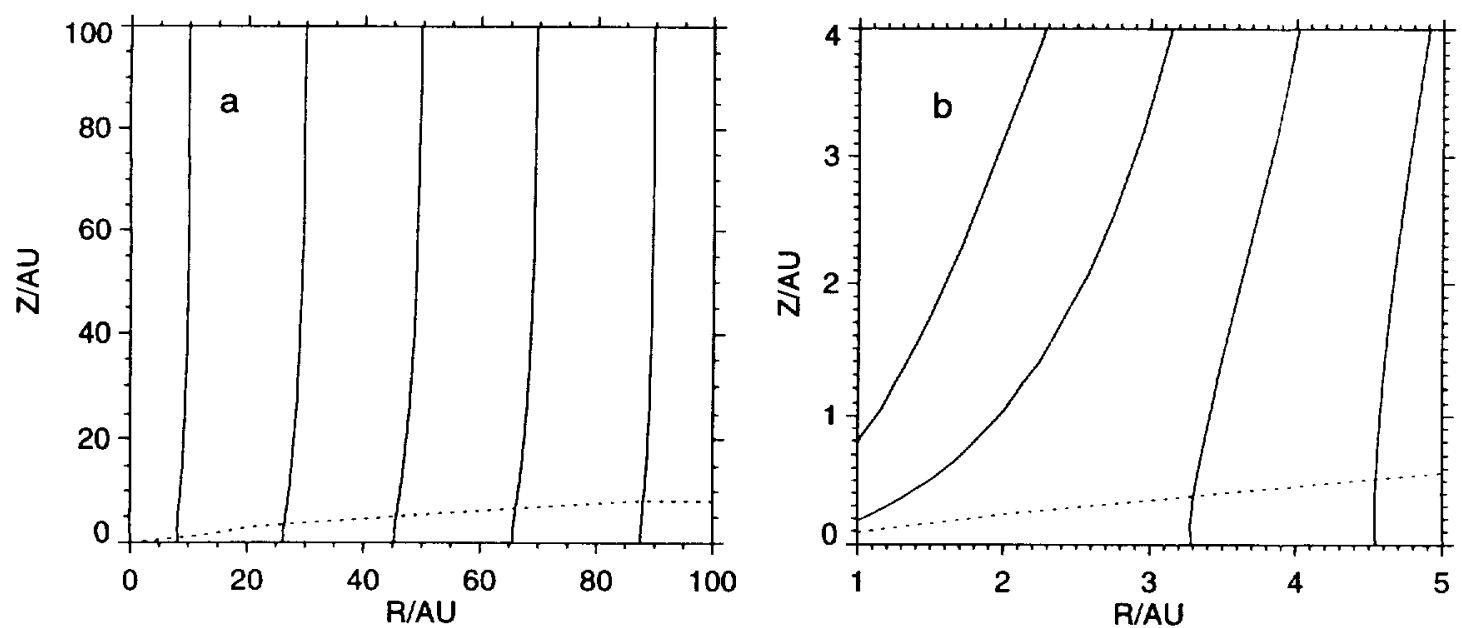

FIG. 4.-Plot of field lines of the steady state poloidal magnetic field resulting from dragging an initially uniform vertical field by the flow in a vi $2 t$ proto-planetary disk characterized by $\mathscr{P}=0.01 ;(a)$ global view, (b) close-up of an inner portion of the disk to show the structure of the poloidal field nes? region of low ionization. 
configuration. These times are all much shorter than the estimated lifetime of proto-planetary disks, which is about $10^{6}-10^{7}$ yr.

Altogether, viscous proto-planetary disks are even less efficient in bending and amplifying an external magnetic field than standard disks. Even in the case of very small $\mathscr{P}$ only the innermost, thermally ionized parts of the disk can support a field geometry capable of launching a centrifugally driven wind. Providing that small values of the magnetic Prandtl number are physically plausible, winds from the inner parts of the disk may be all we need to explain some of the observations. However, as the overwhelming extent of the disk is unable to start a wind, the attractive idea of wind-driven accretion seems not to be an option.

\section{MAGNETIC FIELD DRAGGING IN A PROTO-PLANETARY DISK DESCRIBED BY A PHENOMENOLOGICAL MODEL}

The basic properties of proto-planetary disks inferred from observations are the disk's mass, its size, the accretion rate, and the disk's photospheric temperatures. Radial dependence of photospheric temperatures are well characterized by the power law $T_{\text {eff }}(R) \sim R^{-q}$. The power-law index, $q$, is typically between 0.5 and 0.75 . Viscous disk theory predicts $q=0.75$, but most objects are characterized by a smaller value of $q$. If we assume that the observed spectral energy distribution originates from a disk alone and is not altered by, say, the existence of an additional spherical dusty envelope (Natta 1993), we have to admit the possibility that the disk is powered by a mechanism other than viscous stress. Fitting the power-law radial dependence of photospheric temperatures to observed spectral energy distributions for solar mass stars (Beckwith et al. 1990) one can obtain

$$
T_{\text {eff }}=390\left[\frac{R}{\mathrm{AU}}\right]^{-0.5} \mathrm{~K}
$$

Now we can take an extra step and assume that $T(R) \approx$ $T_{\text {eff }}(R)$. The disk half-thickness can be estimated from hydrostatic equilibrium under the thin disk approximation, $H \approx C_{\mathrm{s}} / \Omega_{\mathrm{K}} \sim R^{5 / 4}$. Adding a radial distribution of surface density is sufficient to fully determine the structure of the disk. Unfortunately, we lack any reliable information about such a distribution. At this stage we take advantage of the so-called minimum-mass solar nebula concept (see, e.g., Hayashi, Nakazawa, \& Nakagawa 1985). Developed for our solar system, it attempts to reconstruct the surface density of the progenitor disk on the basis of the presentday distribution of mass in the solar system. According to this concept, $\Sigma \sim R^{-3 / 2}$. Applying this power law to a proto-planetary disk with a size of $100 \mathrm{AU}$ and a mass of $0.01 M_{\odot}$ we get

$$
\Sigma(R)=920\left[\frac{R}{\mathrm{AU}}\right]^{-1.5} \mathrm{~g} \mathrm{~cm}^{2}
$$

Equations (17) and (18), together with the assumption $T_{\text {eff }} \approx T$, permit calculations of $H$ and $\rho$, thus also computation of the degree of ionization. Such a phenomenological disk is cooler than the viscous disk considered in the previous section; therefore, the inner thermally ionized region is very small. The surface density drops more rapidly with distance from the star, allowing efficient ionization by cosmic rays.
To complete the construction of this model we assume that the disk is accreting with an accretion rate in the range of $\dot{M}=10^{-8}-10^{-6} M_{\odot} \mathrm{yr}^{-1}$ as inferred from observations. We further assume that $\dot{M}$ is radially uniform. The knowledge of $\dot{M}$ fixes the radial velocity of the fiow

$$
V_{R}=-\frac{\dot{M}}{2 \pi R \Sigma}
$$

Generally, radial velocities obtained in this model are significantly larger (especially at large radii) than radial velocities in the viscous proto-planetary disk of comparable $\dot{M}$. The radial velocity expressed in units of the speed of sound is $\left|V_{R} / C_{\mathrm{s}}\right| \approx 10^{-2}\left[\dot{M} /\left(10^{-6} M_{\odot} \mathrm{yr}^{-1}\right)\right](R / A U)$. Thus, for $\dot{M}=10^{-6} \quad M_{\odot} \mathrm{yr}^{-1},\left|V_{R} / C_{\mathrm{s}}\right| \approx 10^{-2}$ at $1 \mathrm{AU}$ and $\left|V_{R} / C_{\mathrm{s}}\right| \approx 1$ at $100 \mathrm{AU}$. In a viscous disk, considered in the previous section, $\left|V_{R} / C_{\mathrm{s}}\right| \approx \alpha(H / R)$, so it remains very small throughout the disk. Turbulence is absent from the model, as its presence is incompatible with the structure of the disk. The phenomenological model describes a well-ionized, swiftly accreting disk, which is ideally suited to drag an external magnetic field.

Figure 5 shows the properties of a magnetic field resulting from dragging the initially uniform field by the gas in the proto-planetary disk described by our phenomenological model. The overall character of the magnetic field is the same for all values of $\dot{M}$ considered. Consider an accretion rate of $\dot{M}=10^{-6} M_{\odot} \mathrm{yr}^{-1}$. In the outer parts of the disk $(R>5 \mathrm{AU})$, where the degree of ionization is high and the radial velocity is fast, the bending of field lines is dramatic. It produces a $B_{R}$ that is larger than $B_{Z}$, the poloidal field is predominantly radial, and the inclination angle, $i$, approaches $90^{\circ}$. The existence of a strong radial component of the magnetic field leads to the generation of an even stronger toroidal field, which dominates the entire magnetic field. In the inner part of the disk $(R<5 \mathrm{AU})$, where the degree of ionization is low and the radial velocity slower, the bending of the magnetic field is inefficient. The relatively strong vertical field there is the result of magnetic field advection from the outer part of the disk. However, because there is no efficient advection within the inner part of the disk, the radial field is very weak there and the inclination angle, $i$, is very small. The toroidal field is insignificant in the inner part of the disk because of very high magnetic diffusivity combined with the small thickness of the disk (see eq. [15]). For smaller values of $\dot{M}$, the character of the magnetic field is the same, but the critical radius that divides the disk into regions of effective and ineffective magnetic field bending is located farther from the star. Figure 6 shows the poloidal magnetic field lines in a disk with $\dot{M}=$ $10^{-6} M_{\odot} \mathrm{yr}^{-1}$.

The time for the magnetic field in a phenomenological model to reach an equilibrium configuration is greater than that for the viscous proto-planetary disk. This follows from the fact that, while field advection is slower for the latter, the distance the field must be dragged in a phenomenological disk is much greater. The time to equilibrium is about $1.6 \times 10^{4}$ yr for the $\dot{M}=10^{-6} M_{\odot} \mathrm{yr}^{-1}$ model, $1.3 \times 10^{5}$ yr for the $\dot{M}=10^{-7} M_{\odot} \mathrm{yr}^{-1}$ model, and $10^{6} \mathrm{yr}$ for the $\dot{M}=10^{-8} M_{\odot} \mathrm{yr}^{-1}$ model.

The structure of a magnetic field in such a protoplanetary disk is markedly different from the structure of a magnetic field in a viscous proto-planetary disk. Bending and amplification of an external magnetic field occur quite 

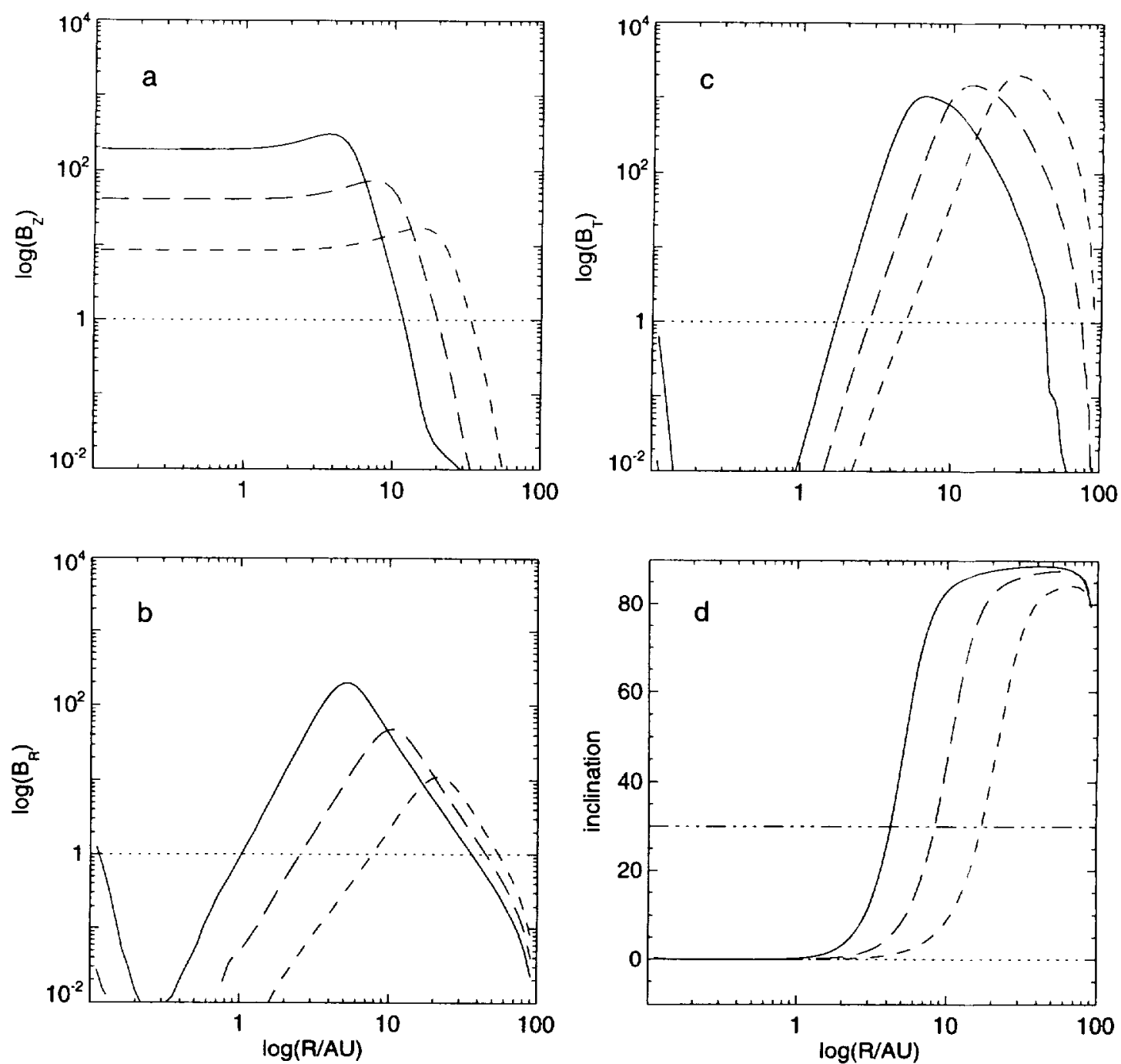

Fig. 5.-Properties of the steady state magnetic field resulting from dragging an initially uniform vertical field by the flow in a phenomenological mod i. a proto-planetary disk. Different line styles correspond to different values of an accretion rate: $\dot{M}=10^{-8} M_{\odot}$ yr ${ }^{-1}$, short-dashed line; $\dot{M}=10^{-7} M$ long-dashed line; $\dot{M}=10^{-6} M_{\odot} \mathrm{yr}^{-1}$, solid line. $(a-c)$ The strength of magnetic fields in a disk as a function of distance from the central star: $(a)$ vertical at the disk's midplane; $(b)$ toroidal field at the vertical location where it achieves its maximum value; $(c)$ radial field at the surface of the disk. The magni i 19 of the magnetic field is measured in units of $B^{\infty}$; the dotted lines, drawn for visual reference, indicate $B=B^{\infty}$. $(d)$ Inclination angle, $i$, between the field $l$ in and the normal to the disk measured at the disk surface, as a function of the distance from the star. Dotted and triple-dot-dashed lines are for visual refert in indicating $i=0$ and $i=30^{\circ}$, respectively.
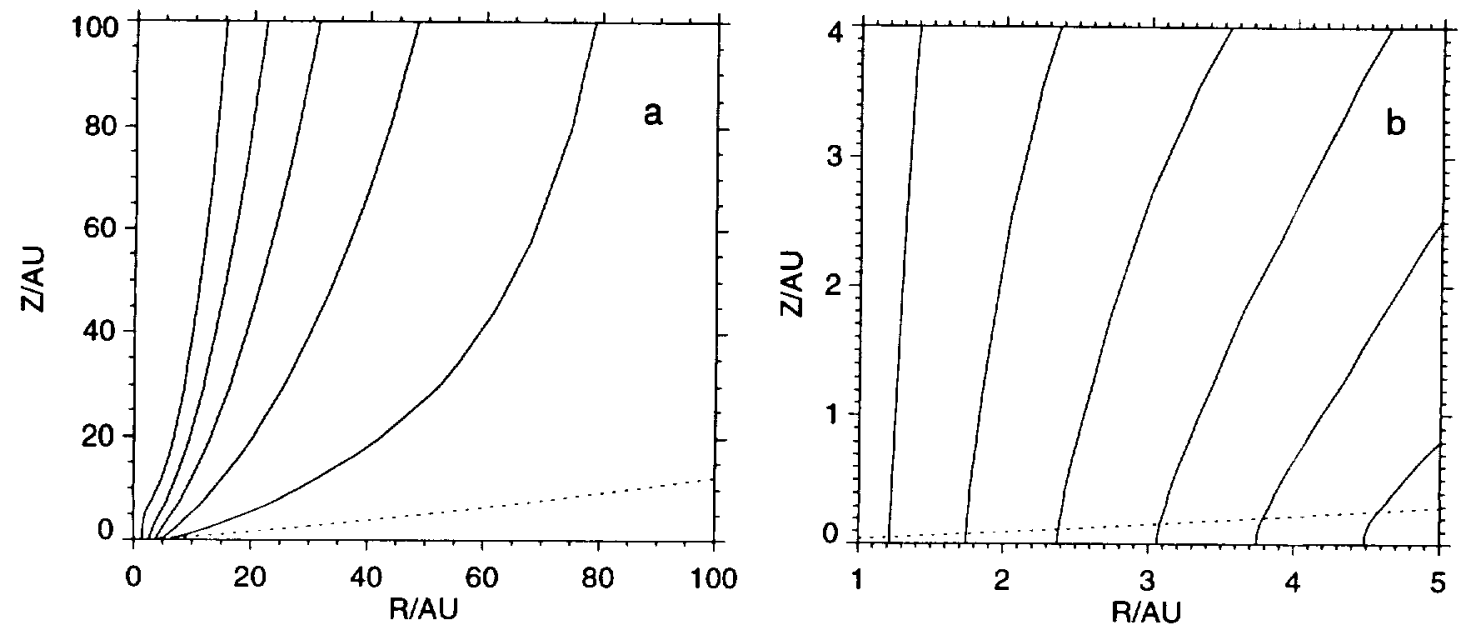

FiG. 6.-Plot of field lines of the steady state poloidal magnetic field resulting from dragging an initially uniform vertical field by the flow i phenomenological model of a proto-planetary disk characterized by $\dot{M}=10^{-6} M_{\odot} \mathrm{yr}^{-1} ;(a)$ global view, (b) close-up of an inner portion of the disk. 
easily. The disk described by a fiducial value of the accretion rate has a wind-launching magnetic field geometry from $R \approx 4 \mathrm{AU}$ outward. Thus, an overwhelming portion of the disk may emit centrifugally driven winds. It is tempting to speculate that our phenomenological disk exemplifies the kind of disks in which the wind is responsible for the accretion flow; hence, high values of $V_{R}$, and an accretion flow, acting on an externally imposed magnetic field, makes the wind possible. This can only be checked by a self-consistent calculation. However, given the basic observational properties of proto-planetary disks, any wind-driven model of a proto-planetary disk must be characterized by fast accretion speed and low surface density.

\section{DISCUSSION}

This paper represents an attempt to study the mechanism of magnetic field dragging in accretion disks in a general and cohesive fashion. This was achieved, albeit only in the kinematic approximation and for disks surrounded by a medium characterized by the magnetohydrodynamical properties of "vacuum." Our objective was twofold; first we aimed at investigating the dragging of magnetic field in viscous disks and, second, we tried to identify a nonviscous accretion disk likely to produce a nontrivial distortion of an externally imposed magnetic field.

The case of a viscous disk is interesting inasmuch as such a disk is, indubitably, the most frequently called-upon concept in accretion disk theory. The turbulence, which is the source of the anomalous viscosity and the cause of the radial movement of the gas that stretches and amplifies the magnetic field, is also the major destroyer of the magnetic field by means of turbulent diffusivity. Thus, in order for the viscous disk to be an effective magnetic field dragger, the turbulence must somehow perform two seemingly irreconcilable tasks: provide high viscosity and weak magnetic diffusivity. Without knowledge about the source and detailed character of the turbulence, its ability to deliver on these two tasks is difficult to assess. Instead, we encapsulate our ignorance about the nature of turbulence into a parameter, the magnetic Prandtl number, that, in our context, provides the measure of the effectiveness of turbulence to destroy the magnetic field relative to its effectiveness to amplify the magnetic field. It is often assumed that $\mathscr{P}$ should be of the order of unity. This is based on two broadly accepted notions regarding turbulent processes. First, turbulent viscosity and the coefficient of turbulent diffusivity are numerically about equal (Canuto \& Battaglia 1988). Second, the turbulent, magnetic diffusivity is numerically about equal to the coefficient of turbulent diffusivity (see Zel'dovich, Ruzmaikin, \& Sokoloff 1983). However, we found that only disks powered by turbulence characterized by small values of $\mathscr{P}$ are capable of bending the external magnetic field to the degree that assures its significant amplification. This conclusion parallels the results of calculations by Lubow et al. (1994a). Is the small $\mathscr{P}$ regime possible? It is useful to recall the meaning of turbulent magnetic diffusivity. The turbulent motion enhance diffusion by mixing together field lines of opposite directions, thus reducing the magnitude of the field and effectively greatly increasing the total magnetic diffusion. At certain magnitude of magnetic field turbulent eddies become ineffective in distorting the field lines and turbulent magnetic diffusion may decrease rapidly, while turbulent viscosity remains unaffected. Thus, in a nonlinear regime, small $\mathscr{P}$ condition cannot be ruled out.
Assuming that $\mathscr{P} \ll 1$ disks exist, the structure of the amplified magnetic field is dominated by its toroidal component. The toroidal component is produced by the Keplerian shear acting on the radial component of the magnetic field. If significant dragging of the poloidal field occurs, the radial component of the magnetic field appears and is distorted by the powerful shear to produce the strong toroidal component. The domination of the toroidal field was not realized by Lubow et al. The magnitude of the toroidal field is inversely proportional to $\alpha$, whereas the magnitude of the poloidal field, in the first approximation, is independent of $\alpha$. In order to assess the importance of strong toroidal field for the overall structure of magnetic field we would have to relax several of our assumptions. The dominant toroidal field controls the nonlinear feedback effects such as $\eta_{t}$ quenching described above. Relaxing the kinematic approximation and considering the selfgeneration mechanisms would result in the poloidal field being affected, and possibly significantly changed by the toroidal field. Relaxing the vacuum boundary conditions could lead to toroidal field presence above the disk surface, further modifying the geometry of the poloidal field. These issues will be addressed in the future work.

In assessing the dynamical importance of the magnetic field in a disk, it is convenient to compare its magnitude to the so-called equipartition value, $B_{\text {eq }}$, given by the balance of magnetic and gas pressures. If a magnetic field is stronger than such an equipartition value, it plays an important, most likely dominant, role in determining the structure of the disk. Both the ratio of the Maxwell stress to the viscous stress and the ratio of the wind torque to the viscous torque are proportional to $\left(B / B_{\text {eq }}\right)^{2}$. For strong fields, $B \geq B_{\text {eq }}$, a kinematic approximation that we adopted in this paper, fails. For our standard disk the equipartition value is given by $B_{\text {eq }} \approx 1.3 \times 10^{3} R_{10}^{-1.3125}$. The radial dependence of a magnetic field amplified by the dragging process is a powerlaw with the value of the power-law index determined by the value of $\mathscr{P}$ and the amplitude determined by the magnitude of the external field, $B^{\infty}$. For values of $\mathscr{P} \approx 1$, the field is dominated by its vertical component, and its radial dependence is much flatter than the radial dependence of $B_{\text {eq. }}$. Magnetic field decreases with radius much slower than $B_{\text {eq }}$. In principle, the disk can be divided into two regimes, the inner disk where $B / B_{\mathrm{eq}}<1$, and the outer disk where $B / B_{\text {eq }}>1$. However, very large values (of the order of $1 \mathrm{G}$ ) of $B^{\circ}$ are needed in order for the outer regime to exist. For small values of $\mathscr{P}$, the field is dominated by its toroidal component, and its radial dependence is much steeper than the radial dependence of $B_{\text {eq }}$. The regime in a disk depends on the value of $B^{\infty}$. If the external field is very small $\left(B^{\infty}<4 \times 10^{-4} \mathrm{G}\right.$ for $\mathscr{P}=0.01$ disk) $B / B_{\text {eq }}<1$ everywhere in a disk and the magnetic field has no dynamical importance. If the external field is larger than some critical value, then disk splits into two parts, the inner part where $B / B_{\text {eq }} \gg 1$ and the outer part where $B / B_{\text {eq }} \ll 1$. In the inner part, a superequipartition field leads to a nonlinear regime in which the magnetic field and disk structure are related in some intricate fashion. In the outer part the magnetic field is dynamically unimportant. Due to the very steep radial dependence of the magnetic field, the portion of the disk with a very strong field is small whereas the portion of the disk with a weak field is large.

It seems that, although small $\mathscr{P}$ disks can provide a field geometry capable of launching a centrifugal wind, the wind 
would have a negligible effect on the dynamics of the overwhelming portion of the disk. There are two possibilities to negate this conclusion. First, if the value of $\mathscr{P}$ is just right, the radial dependence of the amplified magnetic field could be close to the radial dependence of $B_{\mathrm{eq}}$ and the entire disk could be in a single $B / B_{\text {eq }}$ regime. This would happen for $\mathscr{P} \approx 0.03$, for which an inclination angle is just above criti$\mathrm{cal}$ and the wind can be supported. Second, the existence of a strong external magnetic field puts a large portion of the disk in the nonlinear regime, in which the radial dependence of the magnetic field may be less steep than our kinematic approximation indicates.

Overall, launching centrifugal winds from a viscous accretion disk is impossible if we assume the fiducial value of $\mathscr{P} \approx 1$. For $\mathscr{P} \ll 1$, the wind is possible; however, its relevance for disk dynamics is uncertain and highly dependent on values of $\mathscr{P}$ and $B^{\infty}$. Finally, turbulent disks are prone to support the internal generation of a magnetic field such as the MHD dynamo. We plan to address in the future the problem of magnetic field dragging occurring concurrently with the dynamo regeneration process. The resultant magnetic field may have properties altogether different from those calculated in this paper.

The apparent inability of viscous disks to bend the external field so that centrifugally driven wind occurs may be viewed as a disappointment. However, the existence of disks where accretion itself is caused by the wind remains a possibility. Because the problem of wind-powered disks is inherently nonlinear, its treatment is beyond the scope of this paper. We may speculate that some proto-planetary disks are indeed wind-powered. This is because their observational properties can be most easily reconciled with a model of a disk that is characterized by large radial velocities and relatively high-ionization fractions (see $\S 5$ ), features necessary to produce large inclination angles of poloidal field lines. Before embracing such a concept two caveats are in order. First, observational properties of proto-planetary disks could be reconciled as well with a viscous disk model, especially if the disk is surrounded by a thin spherical dusty envelope that scatters starlight back onto the disk and heats the outer parts (Natta 1993), thus accounting for the rela- tively flat radial distribution of disk photospheric to $r$ peratures. Therefore, a swiftly accreting, wind-powered dis: is not the only model capable of explaining the obser vations. Second, and more important, truly self-consist $n$ wind-powered model of a proto-planetary disk has ic been yet constructed. The model we have considered in owes its wind-prone magnetic field geometry to an assur.te distribution of surface density. The fact that the assumpt ${ }_{i} 5$ is based on the concept of the minimum-mass solar neb does not make it any more legitimate (in fact see Stepin $k$ \& Valageas 1995).

Closer examination of the character of the magnetic $f i$ in our phenomenological model of a proto-planetary dis shows the steep radial dependence of the vertical magn $t i$ field in the region where field lines are bent by the flow. Tni resembles the behavior of a magnetic field in the low: standard disk. It means that in order for the kinem: $t$ approximation to be valid, the external field must be sm 11 but then the field in a disk is very weak and its effectiven s in driving an accretion flow is doubtful. This underscote the necessity to construct the nonlinear model encomps: ing disk structure, field dragging, and wind launchi is Lubow et al. (1994b) attempted to construct a simpl example of such a coupled disk model. However, they ii: not calculate self-consistently the structure of the disk. I addition they omitted the toroidal field from considerati in Their solution proved to be unstable. Lovelace, Romano :" \& Newman (1994) constructed a model in which the str :c ture of the disk is calculated, but the dragging of the fiels i not self-consistently taken into account, the inclinatiol angle is assumed constant and uniform, and radial dep $\mathrm{r}$ : dence of magnetic field is taken to accommodate the $\mathrm{s}$ : If similar approach to the wind problem.

This research was done while the authors were suppor 2 by the Lunar and Planetary Institute, which is operated b. USRA under contract No. NASW-4574 with NASA. Thi : i Lunar and Planetary Institute Contribution No. 869. $7 \mathrm{~h}$ work has been partially supported by NASA grant NAG $N$ 3719. M. R. R. acknowledges partial support $f r i n$ CONACyT-Mexico.

\section{REFERENCES}

Balbus, S. A., \& Hawley, J. F. 1991, ApJ, 376, 214

Beckwith, S. V. W. 1994, in Theory of Accretion Disks 2, ed. W. J. Duschl, J. Frank, F. Meyer, E. Meyer-Hofmeister, \& W. M. Tscharnuter (Dordrecht: Kluwer), 1

Beckwith, S. V. W., Sargent, A. I., Chini, R. S., \& Güsten, R. 1990, AJ, 99, 924

Blanford, R. D., \& Payne, D. G. 1982, MNRAS, 199, 883

Canuto, V. M., \& Battaglia, A. 1988, A\&A, 193, 313

Cowling, T. G. 1934, MNRAS, 94,39

Curry, C., Pudritz, R. E., \& Sutherland, P. G. 1994, ApJ, 434, 206

Dubrulle, B. 1992, A\&A, 266, 592 1993, Icarus, 106, 59

Frank, J., King, A., \& Raine, D. 1985, in Accretion Power in Astrophysics (Cambridge: Univ. Press), 75

Hayashi, C., Nakazawa, K., \& Nakagawa, Y. 1985, in Protostars and Planets II, ed. D. C. Black \& M. S. Matthews (Tucson: Univ. Arizona Press) 1100

Horne, K. 1994, in Theory of Accretion Disks 2, ed. W. J. Duschl, J. Frank, F. Meyer, \& E. Meyer-Hofmeister, \& W. M. Tscharnuter (Dordrecht: Kluwer), 77

Königl, A. 1989, ApJ, 342, 208

Lovelace, R. V. E., Romanova, M. M., \& Newman, W. I. 1994, ApJ, 437, 136

Lubow, S. H., Papaloizou, J. C. B., \& Pringle, J. E. 1994a, MNRAS, 267, 235
Lubow, S. H., Papaloizou, J. C. B., \& Pringle, J. E. 1994b, MNRAS, 6 1010

Natta, A. 1993, ApJ, 412, 761

Pelletier, G., \& Pudritz, R. E. 1992, ApJ, 394, 117

Pringle, J. E. 1993, in Astrophysical Jets, ed. D. Burgarella, M. Livic C. P. O'Dea (Cambridge: Cambridge Univ. Press), 1

Pudritz, R. E. 1981, MNRAS, 195, 896

Ray, T. P., \& Mundt, R. 1993, in Astrophysical Jets, ed. D. Burgar M. Livio, \& C. P. O'Dea (Cambridge: Cambridge Univ. Press), 145

Ruden, S. P., \& Pollack, J. B. 1991, ApJ, 375, 740

Shakura, N. J., \& Sunyaev, R. A. 1973, A\&A, 24, 337

Stepinski T. F. 1992, Icarus, 97, 130

Stepinski, T. F., \& Levy, E. H. 1991, ApJ, 379, 343

Stepinski, T. F., Reyes-Ruiz, M., \& Vanhala, H. A. T. 1993, Icarus, 106, I Stepinski, T. F., \& Valageas, P. 1995, in Lunar and Planetary Scis XXVI (Houston: Lunar and Planetary Inst.), 1357

Strom, S. E. \& Edwards, S. 1993, in ASP Conf. Ser. 36, Planets aro $n$ Pulsars, ed. J. A. Phillips, S. E. Thorsett, \& S. R. Kulkarni (San Fi.ar cisco: ASP), 235

Tout, C. A., \& Pringle, J. E. 1992, MNRAS, 259, 602

van Ballegooijen, A. A. 1989, in Accretion Disks and Magnetic Field Astrophysics, ed. G. Belvedere (Dordrecht: Kluwer), 99

Wardle, M., \& Königl, A. 1993, ApJ, 410, 218

Zel'dovich, Y. B. Ruzmaikin, A. A., \& Sokoloff, D. D. 1983, in Magr t Fields in Astrophysics (Dordrecht: Gordon \& Breach), 130 
